\title{
Light is required for conidiation in Aspergillus nidulans
}

\author{
Jeffrey L. Mooney and Lawrence N. Yager \\ Department of Biology, Temple University, Philadelphia, Pennsylvania 19122 USA
}

\begin{abstract}
Light is necessary for asexual sporulation in Aspergillus nidulans but will elicit conidiation only if irradiation occurs during a critical period of development. We show that conidiation is induced by red light and suppressed by an immediate shift to far red light. Conidiation-specific gene functions switch from light-independent to light-dependent activities coincident with the expression of $b r l A$, a regulator of conidiophore development. We also show that light dependence is abolished by a mutation in the velvet gene, which allows conidiation to occur in the absence of light. We propose that the initiation of late gene expression is regulated by velvet and controlled by a red light photoreceptor, whose properties are reminiscent of phytochrome-mediated responses observed in higher plants.
\end{abstract}

[Key Words: Aspergillus nidulans; photoinduced development; conidiation; phytochrome]

Received April 18, 1990; revised version accepted June 18, 1990.

Conidiation (asexual sporulation) in the filamentous ascomycete Aspergillus nidulans involves the formation of multicellular differentiated structures, called conidiophores, which produce pigmented, haploid conidia at precisely scheduled times (Axelrod 1972). The extrinsic environmental conditions required for initiating sporulation in this organism are poorly understood. It has been shown previously that conidiation is strongly inhibited in submerged culture but occurs readily when the mycelia are exposed to an air interface (Morton 1961; Axelrod et al. 1973). The physiological basis for this effect does not appear to involve an osmotic change, because conidiation occurs in surface cultures when the atmosphere is saturated with water (Timberlake and Hamer 1986). Nor does it appear to involve generalized nutrient limitation, because colonies grown on filter paper can be transferred to fresh medium without inhibiting conidiation.

Although the effect of light on sporulation has been documented in a wide variety of fungal species (Tan 1978), there are no known reports of any light-mediated developmental or physiological response affecting asexual development in $A$. nidulans. In this report we show that wild-type $A$. nidulans requires light to conidiate and demonstrate that light-dependent conidiation is determined by the allelic state of the velvet (veA) gene. In addition, we identify a photosensitive period, occurring after the initiation of conidiation and demonstrate that conidiation-specific functions can be classified as light-independent or light-dependent. We also show that some of these light-dependent activities are regulated at the transcriptional level. Finally, we show that conidiation is induced by red light and that this induction is reversed by an immediate shift to far red light, reminiscent of the phytochrome-mediated responses observed in plants.

\section{Results}

Light-dependent conidiation is determined by the allelic state of the veA gene

The original Glasgow wild-type isolate of $A$. nidulans produces weakly conidiating colonies that are overshadowed by a profuse growth of aerial hyphae, giving each colony a velvety appearance. This phenotype is observed when the organism is grown on surface culture in an incubator in which the colonies are periodically exposed to light. Incubation in continuous white light results in the prolific formation of conidiophores bearing abundant, mature conidia. In contrast, incubation in constant darkness results in continued vegetative growth, which does not lead to the formation of reproductive structures (Fig. 1).

The veA1 mutation, which was isolated by Käfer (1965), was adopted as a laboratory standard due to its ability to undergo conidiation without the production of aerial hyphae. This phenotype is observed because the $v e A 1$ mutation abolishes light dependency, allowing conidiation to occur regardless of the presence or absence of light. No difference in phenotype is observed between a conidiating veA1 colony grown either in the light or in the dark and a conidiating $v e A^{+}$colony grown under continuous illumination (Fig. 1).

Genetic linkage of the light-dependent conidiation phenotype to the $v e A$ locus is demonstrated by cosegregation of this phenotype with the wild-type veA allele in heterozygous crosses. Strain WIM 126 (yA2 pabaA1; 


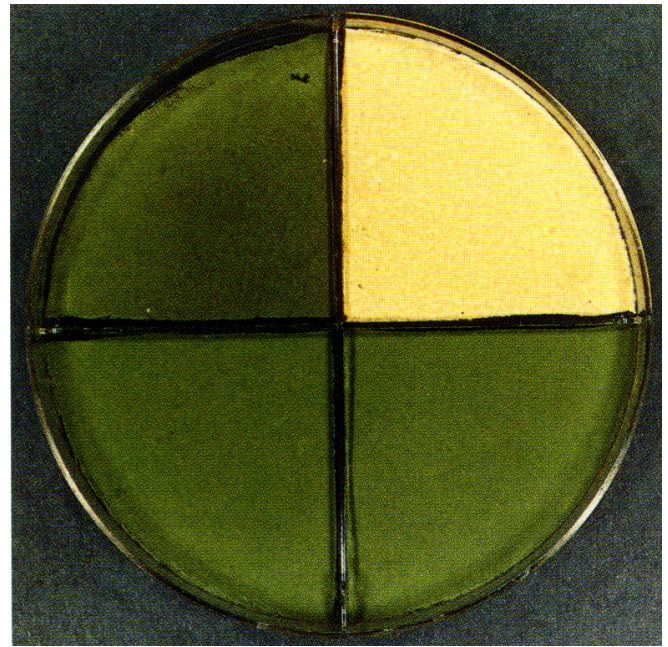

Figure 1. Phenotypes of light- and dark-grown $v e A^{+}$and $v e A 1$ cultures of $A$. nidulans. Confluent lawns of FGSC $4\left(v e A^{+}\right)$or TU 1 (veA1) were incubated for 2 days at $32^{\circ} \mathrm{C}$ either in continuous white light or in complete darkness. Clockwise from upper left: $v e A^{+}$grown in the light; $v e A^{+}$grown in the dark; $v e A 1$ grown in the dark; veA1 grown in the light. Conidiation is evidenced by the green pigmentation found with mature conidia. The dark-grown $v e A^{+}$culture produces only light brown vegetative mycelia.

$v e A^{+}$) was crossed to strain TU 1 (pyroA4; veA1), and the meiotic progeny were scored for the ability to remain aconidial in constant darkness and the presence of aerial hyphae when the colonies were periodically illuminated. The aerial hyphae phenotype, characteristic of the wild-type $v e A^{+}$allele, showed $1: 1$ segregation

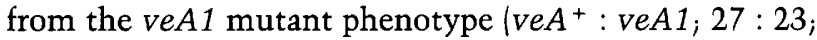
$\left.\mathrm{x}^{2}=0.32 ; \mathrm{df}=1 ; P=0.59\right)$ and assorted independently from $y A 2$, an unlinked spore color mutation $l_{x}^{2}=0.40$; $\mathrm{df}=3 ; P=0.94 \mid$. Every colony that produced aerial hyphae also required light to conidiate, and every colony that had the flat, nonvelvety appearance characteristic of the veA1 mutant conidiated regardless of the presence or absence of light.

The phenotype of a heterozygous diploid, constructed from strains WIM 126 and TU 1, showed that the mutant veA1 allele is recessive to the wild-type $v e A^{+}$allele. As indicated in Table 1, the heterozygous $v e A^{+/ v e A 1}$ diploid conidiated in the light but grew only vegetatively in the dark. A control diploid homozygous for $v e A^{+}($WIM $126 \times$ TU 72) displayed light-dependent conidiation, whereas a homozygous veA1 diploid (TU $1 \times$ FGSC 406) conidiated regardless of illumination.

Because Champe et al. (1981) noted that veA1 abolishes the formation of sexual spores (ascospores) at high temperature $\left(42^{\circ} \mathrm{C}\right)$, the meiotic progeny from the WIM $126 \times$ TU 1 cross were tested for their ability to undergo sexual differentiation at $42^{\circ} \mathrm{C}$. As expected, the light-dependent conidiation phenotype cosegregated with the ability to form viable ascospores at $42^{\circ} \mathrm{C}$; conversely, all veA1 mutant colonies failed to complete sexual development at $42^{\circ} \mathrm{C}$.
As shown in Table 2, the mutational defect of the veA locus does not affect vegetative growth. The submerged growth rate, which is indicative of the mass produced, is not significantly different. In addition, the presence or absence of light has no significant effect on the radial colonial growth rate of either the mutant or the wild type. More important, the veA1 mutation does not affect the time of conidiophore vesicle appearance or the time of mature conidia formation in spore-originated cultures germinated on an agar surface. The veA1 mutation therefore abolishes the requirement that the organism be exposed to light to undergo asexual differentiation without affecting the kinetics of conidiation.

\section{A. nidulans responds to light at a specific period following the initiation of conidiation}

Colonies do not normally conidiate in submerged culture but do if they have acquired competence and are subsequently exposed to an air interface (Axelrod et al. 1973). The transfer of mycelia from liquid to surface culture, termed induction, initiates and synchronizes asexual development (Timberlake 1980). We have shown that competence must be acquired before light can elicit conidiation in induced $v e A^{+}$colonies (J. L. Mooney and L. N. Yager, data not shown). Even after competence, light has no effect on submerged cultures, because a $v e A^{+}$strain grown in liquid medium under continuous illumination, induced, and immediately transferred to the dark will not conidiate. Thus, the period during which $\mathrm{veA}^{+}$colonies must be exposed to light to conidiate can be determined by shifting competent mycelia between light and dark conditions immediately following induction.

The critical period during which wild-type $A$. nidulans must be exposed to light to elicit conidiation is shown in Figure 2. A light pulse given to competent mycelia at any time from 0 to $6 \mathrm{hr}$ after induction results in conidiation, indicating that light is not continuously needed. However, light pulses provided $6 \mathrm{hr}$ or more after induction do not elicit conidiation. When exposed to continuous illumination, $v e A^{+}$colonies produce conidiophore vesicles at $6 \mathrm{hr}$, and mature, viable conidia produce at $12 \mathrm{hr}$ after induction. Thus, wild-type $A$. nidulans responds to light during the critical period defined from the initiation of conidiation (induction) until the initiation of developmental structures.

In Figure 2, induced colonies produced conidia if given

Table 1. Conidial yields in veA diploid strains

\begin{tabular}{lcc}
\hline \multirow{2}{*}{ Genotype } & \multicolumn{2}{c}{ Conidia per colony } \\
\cline { 2 - 3 } & light & dark \\
\hline$v e A^{+/ v e A 1}$ & $4.1 \times 10^{8}$ & $4.3 \times 10^{3}$ \\
$v e A^{+/ v e A^{+}}$ & $3.4 \times 10^{8}$ & $3.1 \times 10^{3}$ \\
$v e A 1 / v e A 1$ & $7.1 \times 10^{8}$ & $4.5 \times 10^{8}$ \\
\hline
\end{tabular}

Colonies were induced, irradiated with $10 \mathrm{~W} / \mathrm{m}^{-2}$ of broad spectrum red light $(610-720 \mathrm{~nm})$, and assayed for conidial yield per colony at $24 \mathrm{hr}$ after induction. 
Table 2. Growth and developmental properties of the veA alleles

\begin{tabular}{|c|c|c|c|c|c|c|c|}
\hline \multirow[b]{2}{*}{ Genotype } & \multirow{2}{*}{$\begin{array}{l}\text { Submerged } \\
\text { growth rate } \\
\left(\mathrm{mg} \text { dry } \mathrm{wt}^{1 / 3} / \mathrm{hr}\right)\end{array}$} & \multicolumn{2}{|c|}{$\begin{array}{l}\text { Radial growth rate } \\
\text { (mm/day) }\end{array}$} & \multicolumn{2}{|c|}{$\begin{array}{l}\text { Time of conidiophore } \\
\text { vesicle appearance } \\
\text { (hr) }\end{array}$} & \multicolumn{2}{|c|}{$\begin{array}{c}\text { Time of conidial } \\
\text { maturation } \\
\text { (hr) }\end{array}$} \\
\hline & & light & dark & light & dark & light & dark \\
\hline$v e A^{+}$ & $0.048 \pm 0.025^{\mathrm{a}}$ & $8.7 \pm 0.4^{b, c}$ & $8.8 \pm 0.3^{\mathrm{c}, \mathrm{d}}$ & 30.0 & - & 37.5 & - \\
\hline$v e A 1$ & $0.088 \pm 0.039^{a}$ & $8.2 \pm 0.2^{b, e}$ & $8.2 \pm 0.8^{\mathrm{d}, \mathrm{e}}$ & 29.5 & 29.5 & 38.0 & 38.0 \\
\hline
\end{tabular}

The strains and complete genotypes used were WIM 126 (yA2 pabaA1; veA $\left.{ }^{+}\right)$and WIM 064 (yA2 pabaA1; veA1).

The time of conidiophore vesicle appearance and the time of conidial maturation were assayed from noninduced single colonies, as described by Axelrod (1972) and Yager et al. (1982).

$n=3$ for the submerged growth rate determination; and $n=5$ for the radial growth rate determination \pm SD. The significance of differences between means was determined by calculating $t$ (Student's $\mathrm{t}$ test, two-tailed).

${ }^{a-e}$ Not significantly different from other values with the same superscript $(P>0.05)$.

a 1-hr exposure to light during the critical period. To determine the minimum exposure time required to elicit conidiation, induced colonies were subjected to the different durations of light shown in Table 3 . These data indicate that between 15 and 30 min of continuous illumination is necessary to elicit conidiation. This minimum exposure time can be provided at any time during the critical period and remains constant during the period of light sensitivity. As described below, conidiation in A. nidulans responds specifically to red light. Although the minimum exposure time was deduced by irradiating induced colonies with broad spectrum red light $\left(610-720 \mathrm{~nm}\right.$ at a fluence rate of $\left.10 \mathrm{~W} / \mathrm{m}^{2}\right)$, no appreciable difference, in comparison to the results shown in Table 3, was observed if white light of the same intensity was substituted.

The minimum intensity required to elicit conidiation is $0.15 \mathrm{~W} / \mathrm{m}^{2}$ at $680 \mathrm{~nm}$. Irradiating $v e A^{+}$colonies with fluence rates between 0.15 and $35 \mathrm{~W} / \mathrm{m}^{2}$ does not affect the minimum exposure time or alter the photosensitive period. However, reciprocity is observed if irradiation occurs beyond the minimum exposure time; with longer light pulses, comparable levels of conidiation are achieved when the product of the fluence rate and exposure time is constant. This result suggests that the amount of energy required to trigger the response is not related to the timing of developmental events. Accordingly, we anticipated exposures of $<30 \mathrm{~min}$ duration not to be cumulative. As expected, three 10-min light pulses provided immediately after induction and separated by 1-hr intervals do not elicit conidiation.

Light-mediated conidiation can be divided into lightindependent and light-dependent functions

The results of the foregoing experiments indicate that wild-type $A$. nidulans must be exposed to light for at least 15-30 min during the critical period, defined from the initiation of conidiation (induction) to the initial formation of differentiated structures. However, it does not necessarily follow that $v e A^{+}$colonies do not initiate any conidiation-specific functions unless exposed to light. It may be the case that induction initiates the activities of certain conidiation-specific genes but that later events, which are involved in the formation of developmental structures, cannot be completed without a light stimulus.

To test this hypothesis, we devised the experiment

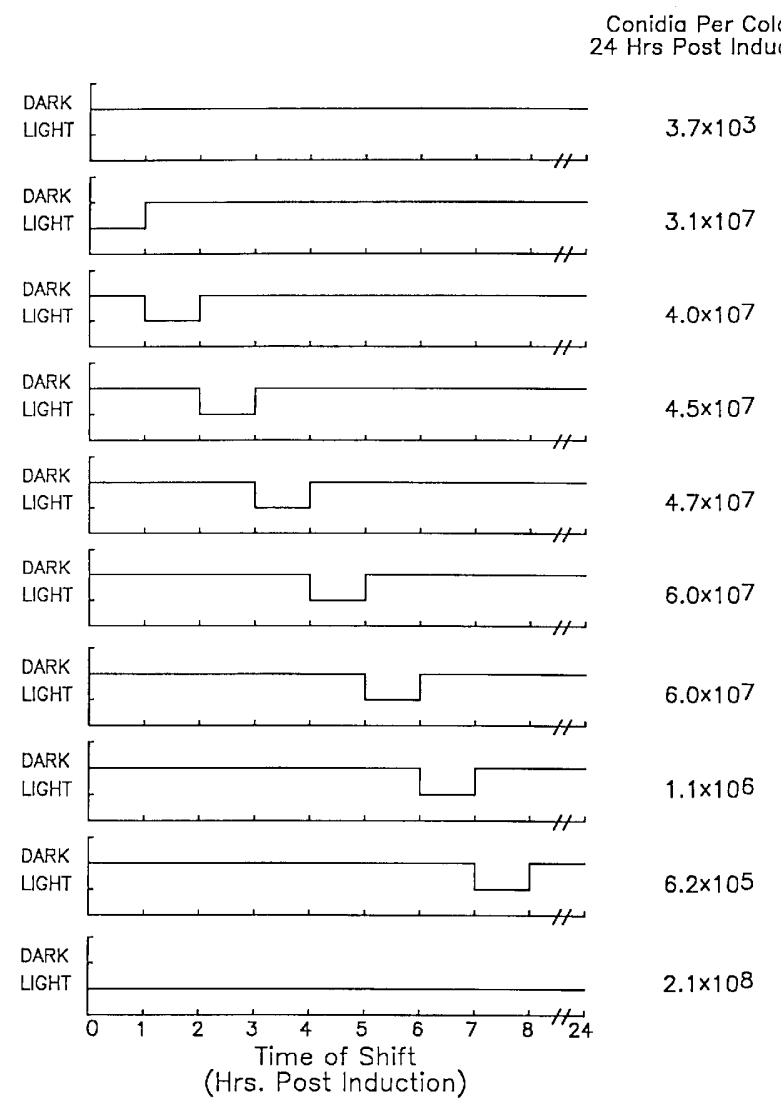

Figure 2. Determination of the critical period of light responsiveness. Single colonies of FGSC 4 were induced and shifted between dark and light conditions, as indicated. Colonies were exposed to white light at an intensity of $10-13 \mathrm{~W} / \mathrm{m}^{2}$ and assayed for conidial yield per colony at $24 \mathrm{hr}$ after induction. The higher than background level of conidia observed with post-6hr shifted colonies is due to conidiation occurring at the circumference of older colonies. The development observed on the outer rim of these colonies represents new growth that is itself responsive to light, whereas the mycelial growth present at the time of induction remains aconidial. 
Table 3. Minimum exposure time required to elicit conidiation

\begin{tabular}{lc}
\hline $\begin{array}{l}\text { Duration of exposure } \\
\text { (hr) }\end{array}$ & Conidia per colony \\
\hline 0.00 & $3.7 \times 10^{3}$ \\
0.25 & $8.1 \times 10^{3}$ \\
0.50 & $4.8 \times 10^{6}$ \\
0.75 & $8.4 \times 10^{6}$ \\
1.00 & $3.1 \times 10^{7}$ \\
2.00 & $3.8 \times 10^{7}$ \\
3.00 & $4.5 \times 10^{7}$ \\
4.00 & $4.9 \times 10^{7}$ \\
5.00 & $6.7 \times 10^{7}$ \\
6.00 & $5.8 \times 10^{7}$ \\
7.00 & $6.6 \times 10^{7}$ \\
24.00 & $2.1 \times 10^{8}$ \\
\hline
\end{tabular}

FGSC $4\left(v e A^{+}\right)$colonies were induced and irradiated with 10 $\mathrm{W} / \mathrm{m}^{-2}$ of broad spectrum red light $(610-720 \mathrm{~nm})$ for the times indicated. Conidial yields were determined $24 \mathrm{hr}$ after induction.

shown in Table 4. The time of conidiophore vesicle appearance was measured after shifting $v e A^{+}$colonies from the dark to the light at various times after induction. Colonies subjected to continuous illumination following induction display a time of conidiophore vesicle appearance at $6 \mathrm{hr}$. We observe that incubation in the dark for as long as $5 \mathrm{hr}$ after induction does not delay the time of vesicle appearance. At $5 \mathrm{hr}$ postinduction, vesicles take only $1 \mathrm{hr}$ to form after colonies are exposed to light. Thus, incubation in the dark following induction does not delay the onset of conidiophore development. Furthermore, if light is not provided to the organism during the critical period, vesicles will never form, because dark-to-light shifts performed after $6 \mathrm{hr}$ postinduction do not result in the formation of differentiated structures or conidia. These results argue that the initia-

Table 4. Effect of delayed exposure to light on developmental kinetics

\begin{tabular}{|c|c|}
\hline $\begin{array}{l}\text { Time of shift to light } \\
\text { (hr postinduction) }\end{array}$ & $\begin{array}{l}\text { Time of conidiophore } \\
\text { vesicle appearance } \\
\text { (hr postinduction) }\end{array}$ \\
\hline 0 & 6 \\
\hline 1 & 6 \\
\hline 3 & 6 \\
\hline 5 & 6 \\
\hline 7 & no vesicles \\
\hline 9 & no vesicles \\
\hline Not shifted & no vesicles \\
\hline \multicolumn{2}{|c|}{$\begin{array}{l}\text { Single colonies of FGSC } 4 \text { were induced, incubated in the dark, } \\
\text { and then shifted to white light at an intensity of } 10-13 \mathrm{~W} / \mathrm{m}^{-2} \\
\text { at the times indicated. Following exposure to light, colonies } \\
\text { were examined microscopically every half hour for the appear- } \\
\text { ance of conidiophore vesicles. Colonies containing } 25 \text { or more } \\
\text { conidiophores were scored as positive, and the time of conidio- } \\
\text { phore vesicle appearance was determined by the method and } \\
\text { criterion of Axelrod et al. (1973). }\end{array}$} \\
\hline
\end{tabular}

tion of conidiation and the expression of some early functions associated with asexual development do not require light but that the expression of those events commencing with the formation of differentiated structures is light dependent.

\section{Light-dependent conidiation-specific genes are transcriptionally regulated}

Induction activates the transcription of a large number of conidiation-specific genes (Timberlake 1980). Because the foregoing analysis suggests that conidiation-specific genes can be classified as light independent or light dependent, one would predict that those genes expressed during the critical period should synthesize transcripts regardless of the presence or absence of light, whereas those genes expressed after the critical period should be unable to accumulate transcripts unless the organism has been exposed to light. We tested this assumption by examining the developmental expression of transcripts encoded by the clones shown in Figure 3 .

Mutations in brlA result in the inability to undergo the transition from conidiophore stalk elongation to vesicle formation and subsequent budding growth (Clutterbuck 1969). The brlA transcript is absent from vegetative cells but accumulates during conidiophore development beginning at about the time of vesicle formation (Johnstone et al. 1985; Boylan et al. 1987). We show that brlA transcripts fail to accumulate in $v e A^{+}$colonies that are grown in complete darkness but do appear at the expected time in colonies exposed to light. brlA transcripts begin to accumulate at $6 \mathrm{hr}$ postinduction in illuminated cultures, not only closely coincident with the initiation of developmental structures but also with the end of the critical period for photoinduction. brlA is therefore the earliest known gene possessing light-dependent gene activity.

$a b a A$ mutants produce normal conidiophores, but the phialides branch and proliferate instead of forming conidia, producing rod-like cells with swellings at intervals (Clutterbuck 1969, 1977). abaA transcripts are absent from vegetative cells but accumulate during conidiophore development beginning at about the time of phialide formation (Boylan et al. 1987). We observe $a b a A$ transcription beginning at $6 \mathrm{hr}$ postinduction in illuminated cultures but lagging behind brlA synthesis in terms of quantitative accumulation, in agreement with the demonstration of Boylan et al. (1987) that brlA precedes $a b a A$ in the developmental pathway. The absence of $a b a A$ transcription in the dark is also consistent with $a b a A$ mRNA accumulation requiring wild-type $b r l A$ expression.

Boylan et al. (1987) isolated a series of cDNA clones, designated pCAN, which showed hybridization to conidiation but not hyphal probes. Transcripts hybridizing to pCAN41 begin to accumulate at the same time as brlA expression but are not expressed in a brlA1 mutant strain. As expected, CAN41 transcription is also light dependent. In contrast, CAN44, CAN65, and CAN77 all accumulate transcripts irrespective of illumination. 


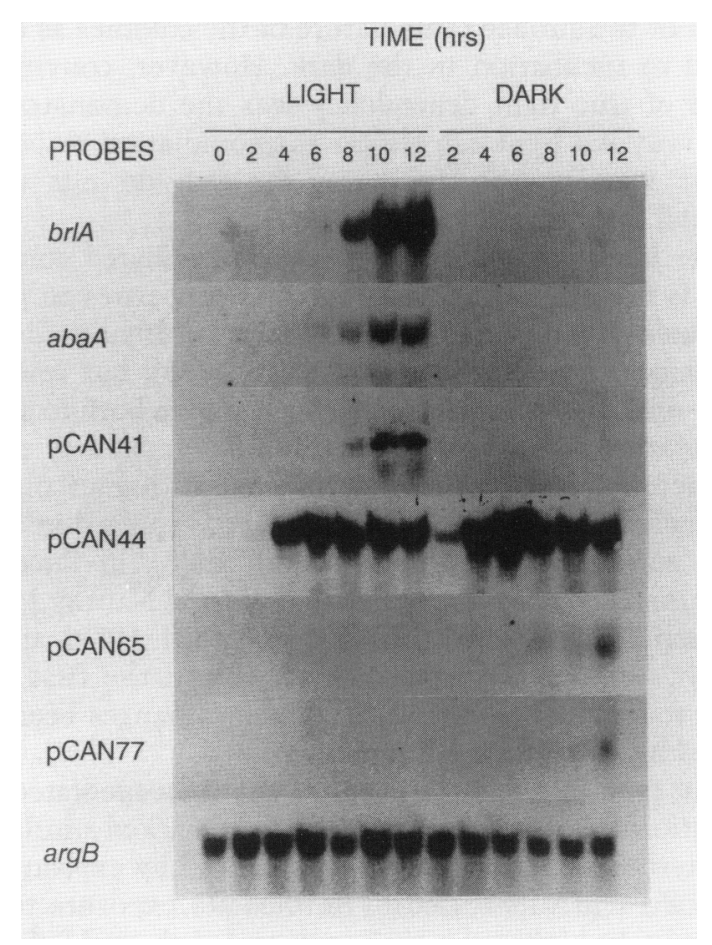

Figure 3. Expression of developmentally regulated transcripts in $v e A^{+}$colonies incubated with and without illumination. Total RNA was isolated from wild-type FGSC 4 colonies at 2-hr intervals following induction. Twenty micrograms of total RNA per lane was fractionated by gel electrophoresis, blotted, and hybridized with the ${ }^{32} \mathrm{P}-1 \mathrm{abeled}$ probes as indicated. The $\arg B$ gene, which is expressed at a constant level throughout development (Yelton et al. 1983), was used as a control. If colonies are exposed to continuous illumination, the following phenotypes are observed with respect to conidiophore development: at $0 \mathrm{hr}$, noninduced undifferentiated hyphae; at $2 \mathrm{hr}$, undifferentiated hyphae; at $4 \mathrm{hr}$, aerial stalks; at $6 \mathrm{hr}$, conidiophore vesicles; at $8 \mathrm{hr}$, metulae and phialides; at $10 \mathrm{hr}$, immature conidia; at $12 \mathrm{hr}$, viable, lightly pigmented conidia. Cultures grown in the dark do not exhibit conidiophore development.

CAN44 and CAN65 initiate transcription just after induction, but barely detectable levels of CAN77 are not observed until the initiation of brlA transcription. All three genes, however, are transcribed in a brlA1 mutant strain (Boylan et al. 1987), suggesting that their activities either precede or function independently of $\operatorname{brlA}$. The transcriptional analysis of CAN65 and CAN77 produced an unexpected result. Both genes show a low level of mRNA accumulation in the light, but a sharp increase in transcription is observed in the dark commencing at $\sim 8 \mathrm{hr}$ after induction for CAN65 and between 10 and 12 hr after induction for CAN77. Because certain conidiation-specific genes function both in asexual and sexual development (Yager et al. 1982; Jurgenson and Champe 1990 ) and the initiation of sexual development is precocious in dark-grown $v e A^{+}$colonies, occurring $\sim 15 \mathrm{hr}$ earlier than in light-grown colonies (J.L. Mooney and L.N. Yager, unpubl.), the late increase in dark-specific transcription of CAN65 and CAN77 may be indicative of different functions utilized during sexual development.

Conidiation in A. nidulans is elicited specifically by red light and reversed by exposure to far red light

The identity of the photoreceptor involved in a lightmediated response may be deduced from an action spectrum of that response. Only those specific wavelengths of light absorbed by the photoreceptor can cause the photochemical change that will elicit a response. An action spectrum shows the relative effectiveness of different wavelengths in eliciting a response and usually corresponds to the absorption spectrum of the photoreceptor (Shropshire 1972). To generate an action spectrum for the light-dependent conidiation response in $A$. nidulans, it was first necessary to obtain a dose-response curve for each tested wavelength. The action spectrum was then calculated by plotting the relative intensity of normalized light necessary to elicit the minimum response against each tested wavelength. To ensure the validity of the action spectrum, the level of the response, in this case the conidial yield per colony, was shown to be a function of total dose (Tan 1978).

The action spectrum for eliciting conidiation in $v e A^{+}$ colonies is shown in Figure 4. Asexual development is maximally sensitive to red light, with a major peak of activity at $680 \mathrm{~nm}$. No conidiation response is observed at wavelengths $<500 \mathrm{~nm}$, even if colonies are subjected to four orders of magnitude more energy than that required to bring about a response at $680 \mathrm{~nm}\left(0.15 \mathrm{~W} / \mathrm{m}^{2}\right)$. Similarly, wavelengths $>710 \mathrm{~nm}$ do not elicit conidiation.

Thus, the action spectrum indicates that light-mediated conidiation in $A$. nidulans is due to activity of a red light photoreceptor. The best characterized regulatory photoreceptor that responds to red light is phytochrome, which is found in higher plants (Pratt 1982;

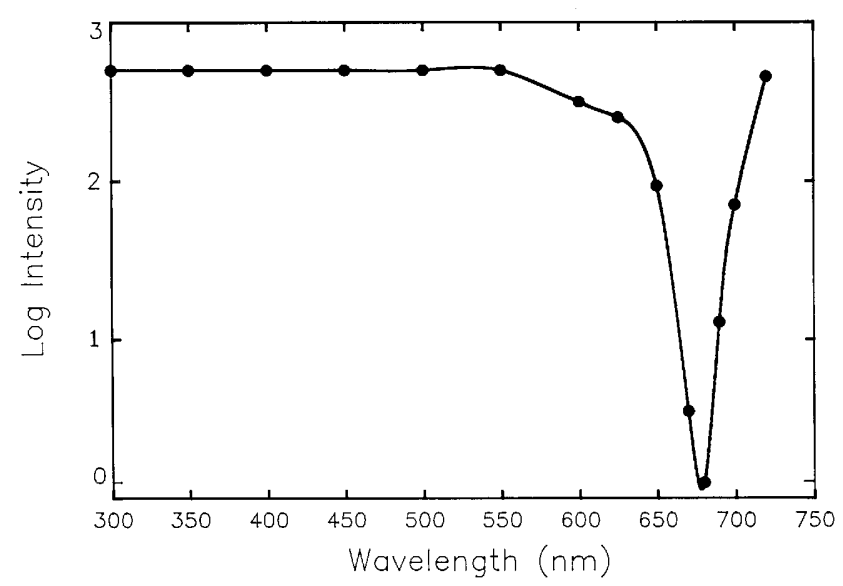

Figure 4. Action spectrum for light-mediated conidiation. The action spectrum, deduced as described in the text, was plotted as the log of the relative intensity of normalized light necessary to elicit the minimum conidial response versus wavelength. 
Silverthorne and Tobin 1987). A major characteristic of phytochrome is its ability to undergo reversible photochemical interconversion between the biologically inactive red light-absorbing form $(\mathrm{Pr})$ and the active far red light-absorbing form (Pfr). The photoconversion of Pr to Pfr by red light induces many developmental and morphogenic responses, including stem elongation and flowering, but exposure to far red light reverses the induction of these responses. The reversal of a red light-induced effect by far red light is therefore a significant and distinguishing feature of the phytochrome photoreceptor.

Table 5 demonstrates that red light-induced conidiation in $\mathrm{veA}^{+}$colonies is reversed by immediate and continuous exposure to far red light $(730 \mathrm{~nm})$. In contrast, colonies given a 1-hr incubation in the dark between a red light to far red light irradiation program do not show reversal of light-induced conidiation. The reversal of red light-induced conidiation by far red light is wavelength specific. Exposure of colonies to green light $1520-570$ $\mathrm{nm}$ at $35 \mathrm{~W} / \mathrm{m}^{2}$ ) does not reverse the effect of red light. Other wavelengths, from 350 to $500 \mathrm{~nm}$, also show no effect. Moreover, exposure of colonies to red light after irradiation with far red light results in conidiation, and return of a far red light-reversed culture to red light also yields conidial colonies.

\section{Discussion}

We have shown that light is required for the formation of conidiophores and viable conidia in $A$. nidulans. While doing the experiments that demonstrated this, we were careful to exclude the possibility that light-dependent conidiation was due to other environmental variables, such as nutrient limitation, changes in oxygen or carbon dioxide levels, gravity, humidity, or temperature. With regard to temperature, we verified that incubation in the light was not altering the temperature of the me-

Table 5. Effect of red and far red irradiation on conidiation

\begin{tabular}{lc}
\hline Irradiation schedule & Conidiation $(\%)^{2}$ \\
\hline Red $(0.5 \mathrm{hr}) \rightarrow$ dark $(24 \mathrm{hr})$ & 100 \\
Dark $(24 \mathrm{hr})$ & $0.5 \pm 0.1$ \\
Far red $(24 \mathrm{hr})$ & $1.2 \pm 0.2$ \\
Red $(0.5 \mathrm{hr}) \rightarrow$ far red $(24 \mathrm{hr})$ & $3.6 \pm 0.8$ \\
Far red $(1-4 \mathrm{hr}) \rightarrow$ red $(0.5 \mathrm{hr}) \rightarrow$ & \\
$\quad$ dark $(24 \mathrm{hr})$ & $51-91^{\mathrm{b}}$ \\
Red $(0.5 \mathrm{hr}) \rightarrow$ far red $(1-4 \mathrm{hr}) \rightarrow$ & \\
$\quad$ red $(0.5 \mathrm{hr}) \rightarrow$ dark $(24 \mathrm{hr})$ & $73-83^{\mathrm{b}}$ \\
\hline
\end{tabular}

FGSC $4\left(\mathrm{veA}^{+}\right)$colonies were induced and irradiated either with $0.5 \mathrm{~W} / \mathrm{m}^{-2}$ of red light $(610-720 \mathrm{~nm})$ or $18 \mathrm{~W} / \mathrm{m}^{-2}$ of far red light $(730 \mathrm{~nm})$ for the times indicated. Percent conidiation was measured at the end of the irradiation schedule by assaying for conidial yield per colony.

aEach assay was normalized to the conidial yield of the $30-\mathrm{min}$ red light to 24 -hr dark control. Each value is the mean of three separate trials $\pm \mathrm{SD}$.

bThe variation in response is due to the time when colonies are exposed to red light following irradiation with far red light. dium or the surface temperature of the colonies as compared to incubation in the dark. However, convincing proof of true light dependency was the demonstration that only red light can trigger the conidiation response. Other wavelengths, including far red, do not elicit asexual development.

The most striking feature of light-mediated conidiation is the similarity of its spectral properties to plant phytochrome. In other fungi, a variety of light-mediated responses have been reported (Tan 1978), but few red light-mediated responses have been shown both to affect sporulation and to display the far red reversibility effect characteristic of phytochrome found in higher plants. Phytochrome-like responses have been reported in Candida guilliermondii (Fraikin et al. 1973), in Puccinia graminis (Lucas et al. 1975, Schneider and Murray 1979), in Verticillium agaricinum (Valadon et al. 1979), and in Botrytis cinerea (Tan 1974), but only in the first case have $\mathrm{red} / \mathrm{far}$ red reversible absorbance changes been detected by in vivo spectrophotometry.

The spectral characteristics of the light-mediated conidiation response in $A$. nidulans bear marked similarity to phytochrome. Conidiation is induced by exposure to red light and suppressed by immediate exposure to far red light. In higher plants the reversal of the red light-induced effect requires immediate irradiation with far red light (Mitrakos and Shropshire 1972). We observe the same effect in $A$. nidulans. Interruption of a red-to-far red irradiation scheme with a period of dark incubation will cancel the effect of far red light and result in conidiation. We have also shown that the biological effect of red light is not manifested unless the duration of exposure is between 15 and $30 \mathrm{~min}$.

Although light-dependent conidiation is highly reminiscent of a phytochrome-mediated response, several of its spectral characteristics are unlike those observed in higher plants. The red light-absorbing form of purified plant phytochrome $(\mathrm{Pr})$ absorbs maximally at $667 \mathrm{~nm}$ (Pratt 1982), whereas the action spectrum for conidiation in $A$. nidulans shows a maximum at $680 \mathrm{~nm}$. Moreover, purified plant phytochrome also has a lesser absorption maximum at blue wavelengths $(300-400 \mathrm{~nm}$; Butler et al. 1964), which is absent in Aspergillus. It must be noted, however, that the $680-\mathrm{nm}$ maximum was obtained by assaying for a biological response from the organism. Thus, it is unclear whether the variation in maximum absorbance is indicative of different red light photoreceptors or whether the higher wavelength and absence of blue response in $A$. nidulans is due to interference from other light-absorbing pigments. On the other hand, both the far red light-absorbing form of purified plant phytochrome (Pfr) and the wavelength of light necessary to reverse red light-induced conidiation show maximum absorbance values at $730 \mathrm{~nm}$.

Most plant phytochromes are characterized by a high irradiance response (Hartmann 1966; Shropshire 1972). Exposure to high-intensity far red light elicits the same response observed with a pulse of red light. We do not observe a high irradiance response in $A$. nidulans, because irradiating induced colonies with $18 \mathrm{~W} / \mathrm{m}^{2}$ of far 
red light for $24 \mathrm{hr}$ does not result in the formation of conidiophores or conidia.

The photoconversion of the Pr form of phytochrome to the Pfr form is extremely rapid and occurs within milliseconds following exposure to red light (Pratt 1982). Colbert et al. (1983) have shown that the levels of translatable phytochrome mRNA decline rapidly after exposure to red light. This decline was detectable within 15-30 min following irradiation and was presumably due to autoregulation of phytochrome gene expression. However, they only needed to expose Avena seedlings to $5 \mathrm{sec}$ of red light to provoke the response. In contrast, between 15 and $30 \mathrm{~min}$ of uninterrupted illumination are necessary to elicit conidiation in $A$. nidulans. A cumulative dosage effect is not observed, because an exposure between 15 and $30 \mathrm{~min}$ is still required even if preceded by exposures of $<15 \mathrm{~min}$. Furthermore, the duration of the minimum exposure time is independent of dose. This period is constant and invariant for colonies exposed to intensities as high as 200 -fold over the lowest dose necessary for conidiation. Following this period, conidial yield becomes a function of dose and reciprocity is observed. However, analogous to the situation observed by Colbert et al. (1983), the minimum exposure time in $A$. nidulans is immediately followed by a short period of sensitivity to reversal by far red light, after which the organism is irreversibly committed to proceeding with conidiation.

The implications of these results are complex but suggest a situation similar to plant phytochrome. The 15- to 30-min period may be required either to regulate the synthesis of the photoreceptor or to control the level of some regulatory factor. Unlike the situation in plants, however, light is continuously required during this period. One possibility is that continuous light may be needed to establish a certain amount of active photoreceptor. Alternatively, specific gene products may not be synthesized until the organism is exposed to light and, thus, are not immediately available for interaction with the photoreceptor. This latter possibility may also explain the period of far red light reversibility that immediately follows the 30 -min exposure to red light. Furthermore, it may be necessary to establish certain regulatory gene interactions to coordinate and control the function of the active photoreceptor. Such interactions are not uncommon in A. nidulans. Mirabito et al. (1989) have proposed a developmental cascade, whereby conidiophore development is regulated by the interactions of $b r l A, a b a A$, and wetA.

Regardless of the identity of the photoreceptor, an important feature of light-mediated conidiation is that $A$. nidulans is responsive to photoinduction only during a specific interval following the initiation of asexual development. To elicit the light-mediated response, the organism must first acquire competence and then be exposed to an inducing stimulus (growth against an air interface). The critical period of light responsiveness begins at the initiation of conidiation, which occurs at induction, and continues until the initial formation of differentiated structures.
Induction initiates a large number of early conidiation-specific gene functions, whose activities are a normal prerequisite for conidiophore development within the organism. The existence of these functions is inferred from the isolation and genetic analysis of aconidial mutants, which are blocked in the formation of developmental structures (Martinelli and Clutterbuck 1971; Yager et al. 1982) and from the molecular analysis of developmental gene regulation (Timberlake 1980). These early functions are followed by late conidiationspecific gene functions, which are involved in the direct formation and regulation of differentiated structures. Several of these late functions have been well characterized (Clutterbuck 1977; Boylan et al. 1987; Adams et al. 1988; Mirabito et al. 1989).

The temporal separation of conidiation-specific genes into early and late functions parallels the division of conidiation-specific genes into light-independent and light-dependent functions deduced from the light/dark shifting analysis. Light is not required for the initiation and function of early conidiation-specific genes but is required for the action of late events that direct conidiophore development. The end of the critical period for light responsiveness agrees closely with the time of the initial appearance of differentiated structures. It is interesting that the switch to light-dependent functions is almost coincident with the expression of brlA. We have shown that the transcription of brlA will not occur unless the organism has been exposed to light sometime during the critical period. Although the repertoire of developmental clones examined is small, we observe lightdependent transcription only for those genes expressed after $b r l A$.

brlA is the earliest known gene defined by mutation that is involved in the direct formation of conidiophores (Clutterbuck 1977). Adams et al. (1988) have demonstrated that $b r l A$ regulates the expression of conidiophore development by showing that the forced misscheduled expression of brlA bypasses all prior conidiationspecific events and is both necessary and sufficient to induce conidiation in vegetative cells. brl $A$ therefore appears to be a possible candidate for controlling the switch to light-dependent functions. The expression of brlA requires both the function of certain light-independent early genes and the completion of light-driven events, which operate through the photoreceptor via a signal transduction pathway.

We have also shown that light-mediated conidiation is determined by the allelic state of the $v e A$ gene. The nature and function of the $v e A$ gene are not yet known. However, one possible interpretation that explains the phenotype of the $v e A 1$ mutation and is consistent with our observations is that the veA gene product is a negative regulator or affects the activity of a negative regulator, which controls the function of conidiation-specific genes. It is feasible that this regulator may act directly on $b r l A$ or may control the activity of a conidiation-specific gene, which precedes $b r l A$ within the asexual reproductive pathway.

The properties and possible function of the $v e A$ gene 
are analogous to the characteristics of the $\operatorname{det} 1$ mutation in Arabidopsis thaliana (Chory et al. 1989). This mutation allows the light-dependent characteristics of wildtype seedlings to be constitutively displayed, regardless of the presence or absence of light. Many of these lightdependent traits, such as the suppression of hypocotyl growth and the expression of the $c a b$ genes, are known to be regulated partly by phytochrome. Chory et al. (1989) propose that the wild-type det 1 gene product acts as a negative regulator or affects the expression of a negative factor, which operates in the signal transduction pathway that couples leaf and chloroplast development to light perception.

We have shown that conidiation in $A$. nidulans is induced by light only if the mycelia are in contact with an air interface and then only for a specific period during early conidial development. In nature, successful spore dissemination requires exposure of the organism to advantageous environmental conditions. It is therefore not surprising that exposure to air and light are two important prerequisites for the induction of asexual development. We have also shown that conidiation is mediated by a red light photoreceptor, whose properties are not unlike phytochrome found in higher plants. Although definitive identification of the photoreceptor will require molecular and biochemical analyses, the results of this study serve as a framework for the elucidation of the complex regulatory pathway, which involves the photoinduction of conidiation-specific gene expression.

\section{Methods}

Aspergillus strains, growth conditions, and genetic techniques

The Glasgow wild-type isolate of $A$. nidulans, FGSC 4 (ve $A^{+}$), and strain FGSC 406 (IuA1 yA2; veA1) were obtained from the Fungal Genetics Stock Center. Strains WIM 064 (yA2 pabaA1; veA1), WIM 065 (wA3; pyroA4; veA1), and WIM 126 (yA2 pabaA1; veA+ | were obtained from S.P. Champe. Strain TU 1 (pyroA4; veA1) was constructed in this laboratory from a meiotic cross of FGSC $4 \times$ WIM 065. Strain TU 72 (1uA1; $v e A^{+}$| was selected from the meiotic progeny of a cross of FGSC $4 \times$ FGSC 406 . Standard $A$. nidulans genetic techniques were used throughout this study (Pontecorvo et al. 1953; Clutterbuck 1974).

The complete growth medium and general culture techniques described by Yager et al. (1982) and Butnick et al. (1984) were used throughout this study. Surface-grown colonies requiring illumination were incubated in Percival incubators (model 1-35LL or 1-30BL) equipped with GE $20-\mathrm{W}$ broad spectrum fluorescent light bulbs positioned at a distance of $20 \mathrm{~cm}$ from the agar surface (average illumination was between 10 and $\left.13 \mathrm{~W} / \mathrm{m}^{2}\right)$. Dark conditions were obtained by wrapping single plates loosely in aluminum foil. Specific wavelengths were generated as described below. All incubations were performed at $32^{\circ} \mathrm{C}$.

Submerged growth rates were determined by harvesting mycelia at timed intervals from $250-\mathrm{ml}$ liquid cultures inoculated with 0.5 conidia/ml. Mycelia were collected by filtration onto washed and desiccated Whatman No. 1 filter paper, which was brought to constant weight. The mycelia were washed twice with $500 \mathrm{ml}$ of deionized water and baked in a vacuum oven at $80^{\circ} \mathrm{C}$ until constant weight was reached $(\sim 6 \mathrm{hr})$. The growth of fungi in submerged culture may obey either a cube-root or exponential relationship (Cocker and Greenshields 1977). Using the growth conditions described, cube-root growth was observed and submerged growth rates were presented as milligrams dry weight ${ }^{1 / 3} / \mathrm{hr}$ (Emerson 1950; Marshall and Alexander 1960). Radial colonial growth rates, which obey a linear growth relationship, were determined as described in Yager et al. (1982).

Unless otherwise indicated, an induction stimulus /transfer from submerged to surface growth), performed $36 \mathrm{hr}$ after inoculation of spores, was used to initiate conidiation. All conidial yield determinations were performed on single colonies grown to a density of $20-30$ colonies per plate. The induction procedure and the method for assaying conidial yield were performed as described by Yager et al. (1982). The time of competence was determined by the methods of Axelrod et al. (1973) and Butnick et al. (1984).

\section{Light/dark shifting experiments}

The light/dark shifting experiments were performed by using the same assay procedure as the temperature-shift experiments described by Yager et al. (1982), except that the incubation temperature was maintained at $32^{\circ} \mathrm{C}$. Colonies were grown submerged for $36 \mathrm{hr}$ before being induced to an air interface and then shifted between light and dark conditions as determined by the experiment. (Thirty-six hours is well past the time of competence, which occurs at $25 \mathrm{hr}$ after inoculation under our experimental conditions.) Conidial yields were determined at $24 \mathrm{hr}$ following induction by harvesting five colonies from each plate and assaying for viable conidia as described above. The appearance of conidiophore vesicles was scored by microscopic examination of colonies by using the method of Axelrod et al. (1973).

\section{RNA preparation, blotting, and hybridization}

Total RNA was isolated from induced single colonies as detailed by Timberlake and Hamer (1986), except that harvested colonies were first lyophilized and then disrupted by grinding. RNA was separated by electrophoresis in formaldehydeagarose gels and transferred without pretreatment to nylon membranes (Duralon-UV, Stratagene) by vacuum blotting. RNA was bound to membranes by UV cross-linking with a Stratagene Stratalinker. Hybridization to ${ }^{32} \mathrm{P}$-labeled probes was according to the procedures recommended by the membrane manufacturer. Plasmids pTA39 (Adams et al. 1988) and pPM11 (Boylan et al. 1987) were used as the $b r l A$ and $a b a A$ gene-specific probes, respectively. The pCAN plasmids have been described (Boylan et al. 1987). Plasmid pMOO6 (Upshall 1986) was used as the $\arg B$ gene-specific probe, which served as a control for all RNA blots. All plasmids were generously provided by W.E. Timberlake.

\section{Action spectrum and far red reversal}

All experiments were performed in a constant temperature room at $32 \pm 1^{\circ} \mathrm{C}$. Conidial yield assays were performed on single colonies, which were exposed to light for $24 \mathrm{hr}$ following induction.

Monochromatic light was generated from a Bausch and Lomb monochrometer ( $500 \mathrm{~mm}$ focal length) equipped with a Phillips $2500 \mathrm{CL}$ tungsten-halogen lamp or GE $1000-\mathrm{W}$ tungsten lamp as a light source. Lenses were used to focus the beam at the entrance slit. Water and/or a heat filter (Balzer Calflex C) intercepted the light beam in front of the entrance slit to reduce 
thermal radiation. Either Kliegal plastic filters, with broad transmission characteristics, or Rosco Laboratories color gelatin filters were used at the exit slit to eliminate second or third order wavelengths. High-intensity illumination conditions were obtained by irradiating the colonies directly with either the tungsten or the tungsten-halogen lamp and using either GAMCOLOR sharp cutoff gelatin filters or Kopp glass filters singly or in combination to intercept the light beam and produce the desired wavelength. Neutral density filters were utilized throughout the experiment to regulate intensity. Intensity was measured by using a Li-Cor Quantum/Radiometer/Photometer equipped with the appropriate sensors.

To determine the effective wavelength of light that elicits a conidial response, all wavelengths were first normalized to an initial quanta of $316.5 \mu \mathrm{E} / \mathrm{m}^{2}$ per sec. Each wavelength was then tested for its ability to induce the minimum conidial response (at least $5 \times 10^{3}$ conidia/colony). Those wavelengths able to elicit this response were then checked to ensure that the law of reciprocity held by checking that the response was a function of total dose (time $\times$ dose rate). Neutral density filters were used to intercept the light beam and to determine the intensity of normalized light that would no longer induce the minimal conidiation response. To produce the action spectrum, the minimum energy required at each wavelength that elicits the desired conidiation response (relative to the initial quanta value) was plotted against wavelength.

The red/far red light reversal experiments were performed on single colonies that were incubated for an additional $2 \mathrm{hr}$ without illuraination following induction. At the end of this period, the irradiation program described in Table 5 was performed. Percent conidiation was determined by assaying for conidial yield per colony, as described above.

\section{Acknowledgments}

We thank Dr. William E. Timberlake for his encouragement and support of this work, for his generous gift of the clones used in this study, and for his many helpful suggestions. We also thank Drs. Laurie Tompkins for critical reading of this manuscript, Richard Waring for thoughtful comments, Thomas Punnett for allowing us to use his facilities to construct the action spectrum, and Mr. Daniel Hassett for technical assistance. This work was supported by a grant from the National Science Foundation (DCB8701897) to L.N.Y.

The publication costs of this article were defrayed in part by payment of page charges. This article must therefore be hereby marked "advertisement" in accordance with 18 USC section 1734 solely to indicate this fact.

\section{References}

Adams, T.H., M.T. Boylan, and W.E. Timberlake. 1988. brlA is necessary and sufficient to direct conidiophore development in Aspergillus nidulans. Cell 54: 353-362.

Axelrod D.E. 1972. Kinetics of differentiation of conidiophores and conidia by colonies of Aspergillus nidulans. J. Gen. Microbiol. 73: 181-184.

Axelrod, D.E., M. Gealt, and M. Pastushok. 1973. Gene control of developmental competence in Aspergillus nidulans. Dev. Biol. 34: 9-15.

Boylan, M.T., P.M. Mirabito, C.E. Willett, C.R. Zimmerman, and W.E. Timberlake. 1987. Isolation and physical characterization of three essential genes from Aspergillus nidulans. Mol. Cell. Biol. 7: 3113-3118.

Butler, W.L., S.B. Hendricks, and H.W. Siegelman. 1964. Action spectra of phytochrome in vitro. Photochem. Photobiol.
3: $521-528$.

Butnick, N.Z., L.N. Yager, T.E. Hermann, M.B. Kurtz, and S.P. Champe. 1984. Mutants of Aspergillus nidulans blocked at an early stage of development secrete an unusual metabolite. J. Bacteriol. 160: 533-540.

Champe, S.P., M.B. Kurtz, L.N. Yager, N.J. Butnick, and D.E. Axelrod. 1981. Spore formation in Aspergillus nidulans: Competence and other developmental processes. In The fungal spore: Morphogenetic controls (eds. G. Turian and H.R. Hohl), pp. 255-276. Academic Press, London.

Chory, J., C. Peto, R. Feinbaum, L. Pratt, and F. Ausubel. 1989. Arabidopsis thaliana mutant that develops as a light-grown plant in the absence of light. Cell 58: 991-999.

Clutterbuck, A.J. 1969. A mutational analysis of conidial development in Aspergillus nidulans. Genetics 63: 317-327.

1974. Aspergillus nidulans. In Handbook of genetics (ed. R.C. King), vol. 1, pp. 447-510. Plenum Press, New York.

- 1977. The genetics of conidiation in Aspergillus nidulans. In Genetics and physiology of Aspergillus (ed. J.E. Smith and J.A. Pateman), pp. 305-317. Academic Press, London.

Cocker, R. and R.N. Greenshields. 1977. Fermenter cultivation of Aspergillus. In Genetics and physiology of Aspergillus (ed. J.E. Smith and J.A. Pateman), pp. 361-390. Academic Press, London.

Colbert, J.T., H.P. Hershey, and P.H. Quail. 1983. Autoregulatory control of translatable phytochrome mRNA levels. Proc. Natl. Acad. Sci. 80: 2248-2252.

Emerson, S. 1950. The growth phase in Neurospora corresponding to the logarithmic phase in unicellular organisms. I. Bacteriol. 60: 221-223.

Fraiken, Y.G., V.N. Verkhoturov, and L.B. Rubin. 1973. Discovery of phytochrome system in yeast Candida guilliermondii. Vestnik. Moskovsk. Univ. Biol. Pochvoved. 5: $54-56$.

Hartman, K.M. 1966. A general hypothesis to interpret "high energy phenomena" of photomorphogenesis on the basis of phytochrome. Photochem. Photobiol. 5: 349-366.

Johnstone, I.I.J., S.G. Hughes, and A.J. Clutterbuck. 1985. Cloning an Aspergillus nidulans developmental gene by transformation. EMBO I. 4: 1307-1311.

Jurgenson, J.E. and S.P. Champe. 1990. The sexual and asexual spores of Aspergillus nidulans contain partially overlapping sets of mRNAs. Exp. Mycol. 14: 89-93.

Käfer, E. 1965. The origin of translocations in Aspergillus nidulans. Genetics 52: 217-232.

Lucas, J.A., R.E. Kendrick, and C.V. Givan. 1975. Photocontrol of fungal spore germination. Plant Physiol. 56: 847-849.

Marshall, K.C. and M. Alexander. 1960. Growth characteristics of fungi and actinomycetes. $J$. Bacteriol. 80: 412-418.

Martinelli, S.D. and A.J. Clutterbuck. 1971. A quantitative survey of conidiation mutants in Aspergillus nidulans. $J$. Gen. Microbiol. 69: 261-268.

Mirabito, P.M., T.H. Adams, and W.E. Timberlake. 1989. Interactions of three sequentially expressed genes control temporal and spatial specificity in Aspergillus development. Cell 57: 859-868.

Mitrakos, K. and W. Shropshire, Jr., eds. 1972. Phytochrome. Academic Press, New York.

Morton, A.G. 1961. The induction of sporulation in mould fungi. Proc. R. Soc. Lond. Biol. Sci. 153: 548-569.

Pontecorvo, G., J.A. Roper, L.M. Hemmons, K.D. Macdonald, and A.W.J. Bufton. 1953. The genetics of Aspergillus nidulans. Adv. Genet. 5: 141-238.

Pratt, L.H. 1982. Phytochrome: The protein moiety. Annu. Rev. 
Plant Physiol. 33: 557-582.

Schneider, M.J. and B.J. Murray. 1979. Phytochrome mediation of uredospore germination in the fungus Puccinia graminis. Photochem. Photobiol. 29: 1051-1052.

Shropshire, W. Jr. 1972. Action spectroscopy. In Phytochrome (ed. K. Mitrakos and W. Shropshire, Jr.), pp. 161-181. Academic Press, New York.

Silverthorne, J. and E.M. Tobin. 1987. Phytochrome regulation of nuclear gene expression. Bioessays 7: 18-23.

Tan, K.K. 1974. Red-far-red reversible photoreaction in the recovery from blue light inhibition of sporulation in Botrytis cinera. J. Gen. Microbiol. 82: 201-202.

- 1978. Light-induced fungal development. In The filamentous fungi (ed. J.E. Smith and D.R. Berry), vol. 3, pp. 334-357. Halsted Press, New York.

Timberlake, W.E. 1980. Developmental gene regulation in Aspergillus nidulans. Dev. Biol. 78: 497-510.

Timberlake, W.E. and J.E. Hamer. 1986. Regulation of gene activity during conidiophore development in Aspergillus nidulans. In Genetic engineering (ed. J.K. Setlow and A. Hollaender|, pp. 1-29. Plenum Press, New York.

Upshall, A. 1986. Genetic and molecular characterization of $\arg B^{+}$transformants of Aspergillus nidulans. Curr. Genet. 10: $593-599$.

Valadon, L.R.G., M. Osman, and R.S. Mummery. 1979. Phytochrome mediated carotenoid synthesis in the fungus Verticillium agaricinum. Photochem. Photobiol. 29: 605-607.

Yager, L.N., M.B. Kurtz, and S.P. Champe. 1982. Temperatureshift analysis of conidial development in Aspergillus nidulans. Dev. Biol. 93: 92-103.

Yelton, M.M., J.E. Hamer, E.R. de Souza, E.F. Mullaney, and W.E. Timberlake. 1983. Developmental regulation of the Aspergillus nidulans trpC gene. Proc. Natl. Acad. Sci. 80: $7576-7580$. 


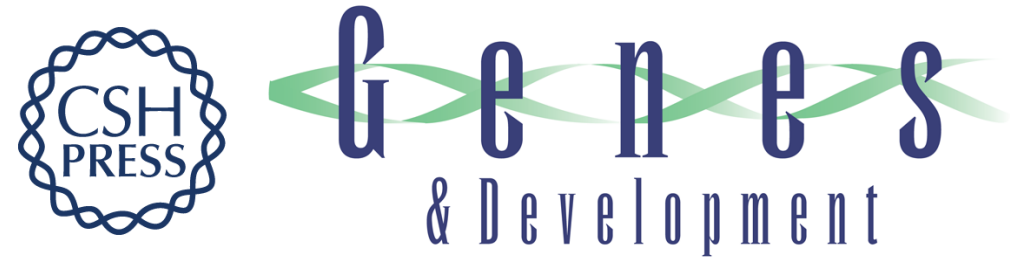

\section{Light is required for conidiation in Aspergillus nidulans.}

J L Mooney and L N Yager

Genes Dev. 1990, 4:

Access the most recent version at doi:10.1101/gad.4.9.1473

References This article cites 30 articles, 9 of which can be accessed free at:

http://genesdev.cshlp.org/content/4/9/1473.full.html\#ref-list-1

License

Email Alerting

Receive free email alerts when new articles cite this article - sign up in the box at the top Service right corner of the article or click here.

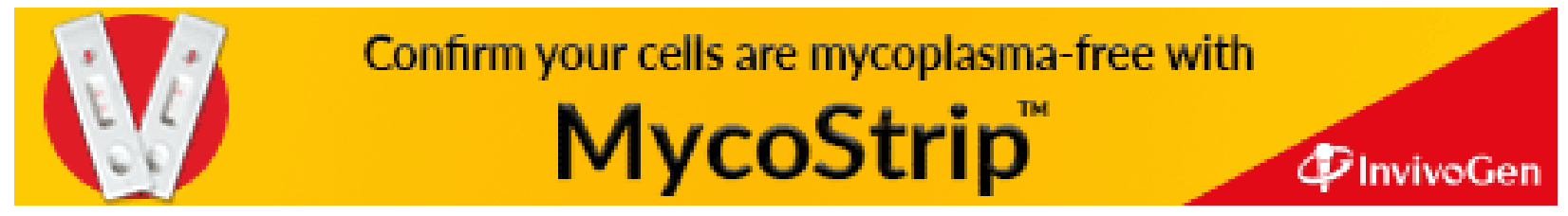

